\title{
Supporting Information for Readers
}

\section{Bulk-type all solid-state batteries with $5 \mathrm{~V}$ class $\mathrm{LiNi}_{0.5} \mathrm{Mn}_{1.5} \mathrm{O}_{4}$ cathode and $\mathrm{Li}_{10} \mathrm{GeP}_{2} \mathrm{~S}_{12}$ solid electrolyte}

Gwangseok Oh, Masaaki Hirayama*, Ohmin Kwon, Kota Suzuki, and Ryoji Kanno

Tokyo Institute of Technology, G1-1, 4259, Nagatsuda, Midoriku, Yokohama, 226-8502, Japan

\section{S1. Electrochemical properties of 7.9 $\mu \mathrm{m}$ LNMO particles}

A composite cathode of LNMO/LGPS was fabricated with LNMO powder (average particle size of $7.9 \mu \mathrm{m}$ and surface area of $0.62 \mathrm{~m}^{2} \mathrm{~g}^{-1}$ ). The LNMO particles were coated by $3.5 \mathrm{wt} . \%$ $\mathrm{LiNbO}_{3}$. Figure $\mathrm{S} 1 \mathrm{a}$ shows the SEM/EDX images of $\mathrm{LiNbO}_{3}$-coated $\mathrm{LNMO}$. The $\mathrm{Nb}$ atoms are homogeneously distributed on the LNMO particles. The coating condition of $\mathrm{LiNbO}_{3}$ on the 7.9 $\mu \mathrm{m}$ LNMO particles is similar to that on the $9.5 \mu \mathrm{m}$ LNMO particles as shown in Fig. 1. All solid-state battery was fabricated with LGPS and Li metal as solid electrolyte and anode, respectively, under the same procedures with $9.5 \mu \mathrm{m}$ LNMO/LGPS/Li. Figure S1b shows the charge-discharge curves of $\mathrm{LiNbO}_{3}$-coated LNMO. The $7.9 \mu \mathrm{m}$ LNMO exhibits the initial discharge capacity of $93 \mathrm{mAh} \mathrm{g}^{-1}$, which is higher than that of $9.5 \mu \mathrm{m}$ LNMO. The contact area between the LNMO and the LGPS increases by decreasing size of the LNMO particles, which enhances the electrochemical reaction. In contrast, the discharge capacities gradually decreased to $55 \mathrm{mAh} \mathrm{g}^{-1}$ at tenth cycle. No significant improvement of cycle stability is observed for the small size LNMO particles, which is indicative of that the capacity fading is independent on the contact 
(a)
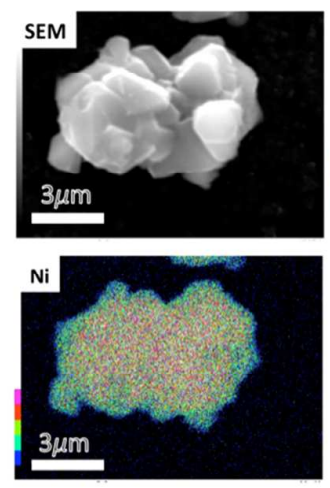
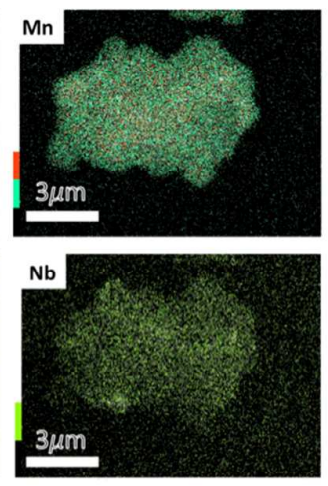

(b)

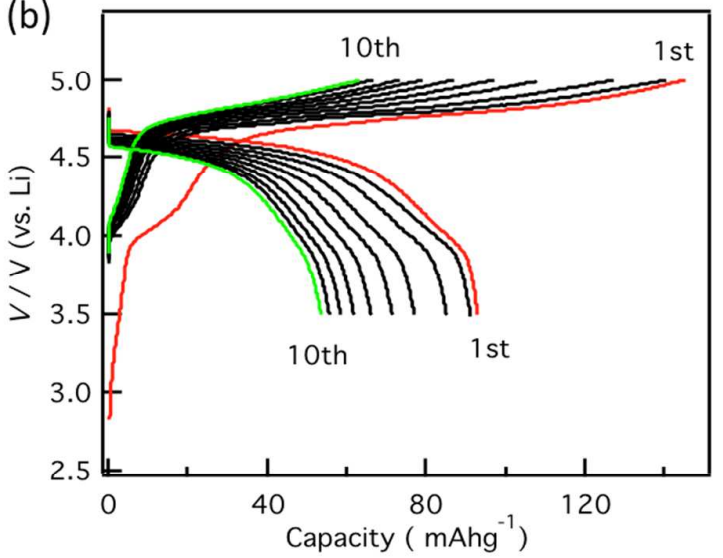

Figure S1. (a) SEM and EDS images $\mathrm{LiNbO}_{3}$-coated $7.9 \mu \mathrm{m}$ LNMO particles and (b) charge-discharge curves of $\mathrm{LiNbO}_{3}$-coated $7.9 \mu \mathrm{m}$ LNMO cathode. The current density used was $7.3 \mathrm{~mA} \mathrm{~g}^{-1}(0.05 \mathrm{C})$.

\section{S2. Procedures of EIS analyses}

EIS spectra of symmetric cells of LNMO/LGPS/LNMO, Li-In/LGPS/Li-In, and Li/LGPS/Li were collected to identify the reaction elements shown in Figure 3(a-c). Prior to the EIS measurements, the LNMO/LGPS/LNMO cell was charged at a current density of $14 \mathrm{~mA} \mathrm{~g}^{-1}$ for 20 min, which corresponded to a charge capacity of $4.7 \mathrm{mAh}^{-1}$. The $\mathrm{Li} / \mathrm{LGPS} / \mathrm{Li}$ and $\mathrm{Li}-\mathrm{In} / \mathrm{LGPS} / \mathrm{In}-\mathrm{Li}$ cells were also charged under the same conditions as the LNMO/LGPS/LNMO cell. The LNMO/LGPS/LNMO cell indicates that the characteristic frequency and the capacitance at the cathode/electrolyte interface are around $30 \mathrm{~Hz}$ and $10^{-6} \mathrm{~F}$, respectively. The Li-In/LGPS/Li-In, and $\mathrm{Li} / \mathrm{LGPS} / \mathrm{Li}$ cells indicates that the characteristic frequency and the capacitance at the anode/electrolyte interface are around $1.5 \mathrm{~Hz}$ and $\sim 10^{-3} \mathrm{~F}$, respectively, which corresponds to the electrochemical reaction at the anode surface. The Li/LGPS/Li cell shows another semicircle at the characteristic frequency of $\sim 10^{-8} \mathrm{~Hz}$, which corresponds to a SEI layer (See section S3). The capacitance and resistivity values of each component were refined by fittings of the ac impedance 
data using equivalent circuits models as shown in Figure $S 2 . \mathrm{R}_{B}$ is bulk resistance of LGPS, $\mathrm{R}_{\mathrm{SEI}}$ and $\mathrm{CPE}_{\mathrm{SEI}}$ is reaction resistance and constant phase element for the $\mathrm{SEI}$ layer, $\mathrm{Rc}$ and $\mathrm{C}_{\mathrm{C}}$ is reaction resistance and constant phase element for the LGPS/LNMO interface, $R_{A}$ and $C_{A}$ is reaction resistance and constant phase element for anode/LGPS interface. The fitting results are summarized in Table S1. In this study, we report unnormalized capacitance values (in F) to identify each component because it is difficult to determine the effective surface areas of LNMO/LGPS, LGPS/Li, and LGPS/In-Li.

(a)

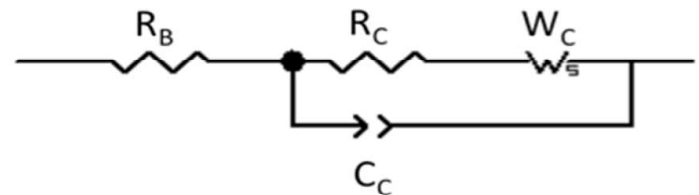

(b)

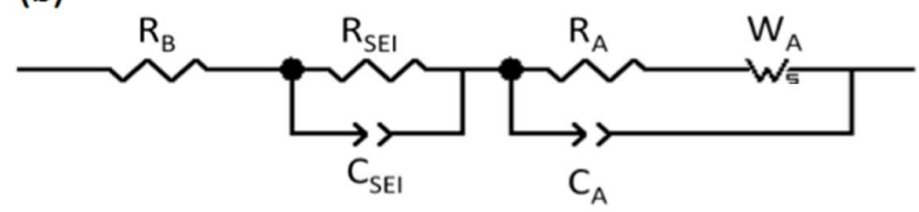

Figure S2. Equivalent circuit models for (a) LNMO/LGPS/LNMO and Li-In/LGPS/Li-In and (b) Li/LGPS/Li symmetric cells.

Table S1 Fitting results of EIS analyses for LNMO/LGPS/LNMO, Li-In/LGPS/Li-In, and Li/LGPS/Li symmetric cells.

(i) cathode side

\begin{tabular}{cccc}
\hline & $\mathbf{R}_{\mathrm{B}} / \boldsymbol{\Omega}$ & $\mathbf{R}_{\mathrm{C}} / \mathbf{\Omega}$ & $\mathbf{C}_{\mathrm{C}} / \mathbf{F}$ \\
\hline LNMO/LGPS/LNMO & 254 & 3883 & $8.9 \times 10^{-6}$
\end{tabular}

(ii) anode side

\begin{tabular}{cccccc}
\hline & $\mathbf{R}_{\mathrm{B}} / \boldsymbol{\Omega}$ & $\mathbf{R}_{\mathrm{A}} / \boldsymbol{\Omega}$ & $\boldsymbol{C}_{\mathrm{A}} / \mathbf{F}$ & $\boldsymbol{R}_{\mathrm{SEI}} / \boldsymbol{\Omega}$ & $\boldsymbol{C}_{\mathrm{SEI}} / \mathbf{F}$ \\
\hline In-Li/LGPS/In-Li & 65 & 49 & $2.9 \times 10^{-3}$ & & \\
\hline Li/LGPS/Li & 130 & 177 & $1.8 \times 10^{-3}$ & 59 & $1.2 \times 10^{-8}$ \\
\hline
\end{tabular}


In this study, we analyzed the EIS results of the LNMO/LGPS/Li battery charged at $4.6 \mathrm{~V}$.

We constructed a fitting model based on the EIS results for the symmetric cells charged at a current density of $14 \mathrm{~mA} \mathrm{~g}^{-1}$ for $20 \mathrm{~min}$. The state-of-charge were different between the cells. To confirm that the characteristic frequency was independent of the charge-of-state, EIS spectra obtained at different cell voltages were analyzed. Figure S3 shows the impedance spectra and fitting curves of the LNMO/LGPS/Li battery at different voltages during the first cycle. Table 2 summarizes the resistance and capacitance values of each component. The observed semicircles have similar capacitance values $\left(\sim 10^{-5} \mathrm{~F}\right)$, which are independent of the cell voltage. Thus, we have identified the appropriate reaction component and have discussed cycle dependence using the EIS data collected at a voltage of $4.6 \mathrm{~V}$.

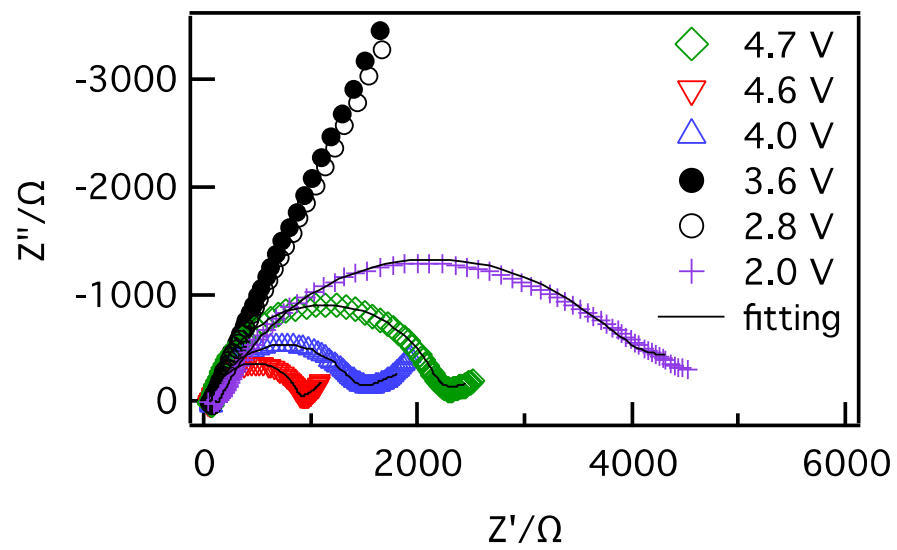

Figure S3. Nyquist plots of the LNMO/LGPS/Li cell at 2.0, 2.8, 3.6, 4.0, 4.6, and $4.7 \mathrm{~V}$ (vs. $\left.\mathrm{Li}^{+} / \mathrm{Li}\right)$.

Table S2. Fitting results for the Nyquist plots corresponding to the LNMO/LGPS/Li cell at 2.0, 2.8, 3.6, 4.0, 4.6, and 4.7 V (vs. $\left.\mathrm{Li}^{+} / \mathrm{Li}\right)$.

\begin{tabular}{ccccccc}
\hline Cell & Voltage & $\mathrm{R}_{\mathrm{B}} / \Omega$ & $\mathrm{R}_{\mathrm{SEI}} / \Omega$ & $\mathrm{C}_{\mathrm{SEI}} / \mathrm{F}$ & $\mathrm{R}_{\mathrm{E}} / \Omega$ & $\mathrm{C}_{\mathrm{E}} / \mathrm{F}$ \\
\hline \multirow{3}{*}{ LNMO/LGPS/Li } & $2.0 \mathrm{~V}$ & 74 & 50 & $9.52 \times 10^{-8}$ & $3.7 \times 10^{3}$ & $8.5 \times 10^{-5}$ \\
\cline { 2 - 7 } & $4.0 \mathrm{~V}$ & 20 & 30 & $5.62 \times 10^{-8}$ & $1.3 \times 10^{3}$ & $3.1 \times 10^{-5}$ \\
\cline { 2 - 7 } & $4.6 \mathrm{~V}$ & 64 & 51 & $8.90 \times 10^{-8}$ & $0.8 \times 10^{3}$ & $1.5 \times 10^{-5}$ \\
\cline { 2 - 7 } & $4.7 \mathrm{~V}$ & 51 & 22 & $8.78 \times 10^{-8}$ & $2.1 \times 10^{3}$ & $2.5 \times 10^{-5}$ \\
\hline
\end{tabular}




\section{S3. XRD patterns of the anode/LGPS interfaces after tenth cycles}

For a further understanding of the interfacial reactions during the electrochemical cycling, ex situ XRD measurements were performed for the LNMO/LGPS/In-Li and LNMO/LGPS/Li batteries. Figure S4 shows XRD patterns of Li/LGPS and In-Li/LGPS interfaces after the tenth cycle. The Li-In/LGPS interface showed diffraction peaks attributed to the Li-In electrode and the LGPS solid electrolyte. No other diffraction peaks were observed after the tenth cycle. In contrast, the Li/LGPS interface showed very weak diffraction peaks of the LGPS phase. Broad diffraction peaks appeared at $26.7,31.6,44.1$ and $52.2^{\circ}$, which could be identified to $\mathrm{Li}_{2} \mathrm{~S}$.

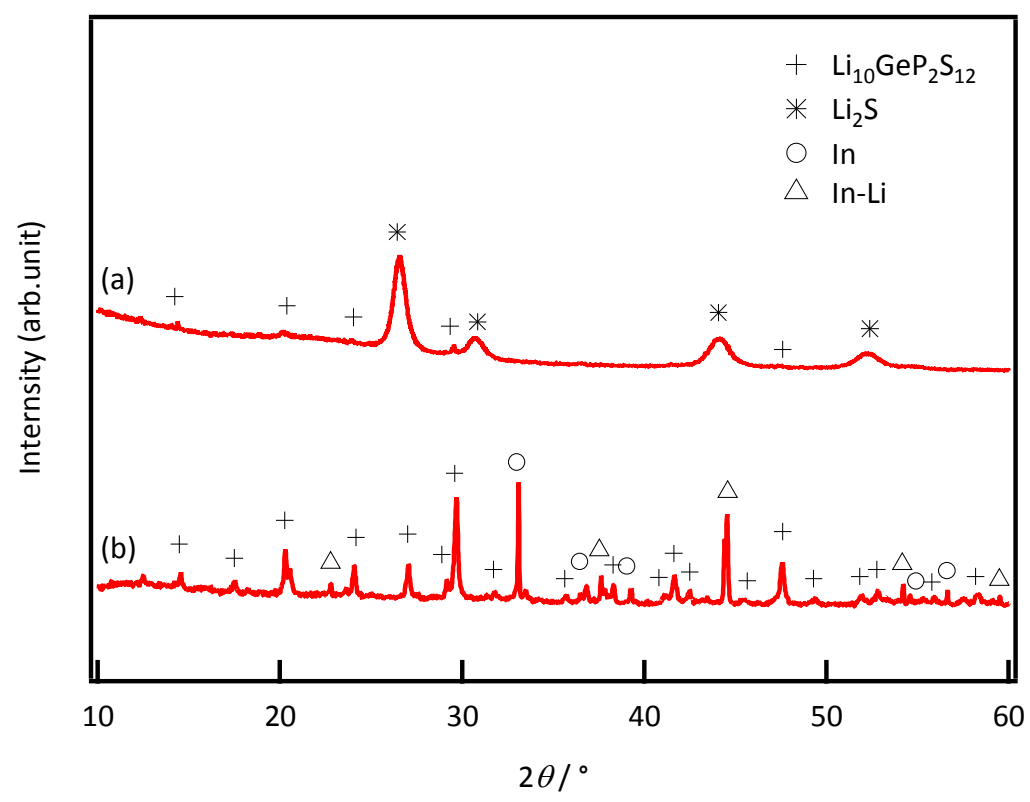

Figure S4. XRD patterns of (a) Li/LGPS and (b) In-Li/LGPS interface after 10 cycles. Diffraction peaks are identified based on ICSD data of $\mathrm{Li}_{10} \mathrm{GeP}_{2} \mathrm{~S}_{12}$ [No. 248307], $\mathrm{Li}_{2} \mathrm{~S}$ [No. 54396], In[No. 182786], and In-Li[No. 51959]. 